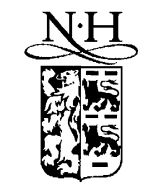

ELSEVIER

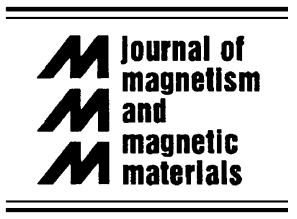

www.elsevier.com/locate/jmmm

\title{
Fast switching in single-domain particles with uniaxial quartic anisotropy
}

\author{
Jyh-Shinn Yang ${ }^{\mathrm{a}, *}$, Ching-Ray Chang ${ }^{\mathrm{b}}$ \\ ${ }^{a}$ Institute of Optoelectronic Science, National Taiwan Ocean University, 2 Pei-Ning Road, Keelung 20224, Taiwan \\ ${ }^{\mathrm{b}}$ Department of Physics, National Taiwan University, Taipei 106, Taiwan
}

Available online 30 October 2004

\begin{abstract}
The effect of quartic anisotropy on angular dependence of the high-speed switching behavior for uniaxial particles is studied. For large applied field angles with respect to the anisotropy axis, the magnetization reversal is dominated by the precessional switching and the switching speed is very fast in the presence of quartic anisotropy. The quartic anisotropy enhances the switching fields moderately, and expands the precessional switching regime towards the anisotropy axis, while slightly increases the switching time for the applied field magnitude larger than the anisotropy field. The angular dependence of switching field exhibits a broad minimum around the field angle of $45^{\circ}$ for large quartic anisotropy. The results have implications for improving the recording characteristics of the tilted media.
\end{abstract}

(C) 2004 Elsevier B.V. All rights reserved.

PACS: $75.40 . \mathrm{Gb} ; 75.40 \mathrm{Mg} ; 75.50 . \mathrm{Ss}$

Keywords: Quartic anisotropy; Fast switching; Precessional switching; Tilted media

\section{Introduction}

In recent years, the storage density and data transfer rate in magnetic recording are increasing very rapidly. The recording areal density beyond $1 \mathrm{Tbit} / \mathrm{in}^{2}$ and data rates beyond $1 \mathrm{Gbit} / \mathrm{s}$ were forecasted for perpendicular magnetic recording $[1,2]$. However, the achievable data density will be

\footnotetext{
*Corresponding author. Tel.: + 886224622192 x 6706; fax: +886224634360 .

E-mail address: jyang@mail.ntou.edu.tw (J.-S. Yang).
}

restricted by the fundamental physical phenomena such as the thermal instability and the switching speed of magnetic domain. To achieve an extremely high recording density, the size of magnetic grain should be accordingly scaled down to maintain a high signal-to-noise ratio (SNR). The reduction in grain size deteriorates the thermal stability owing to superparamagnetism. One approach to improve the thermal stability is to enhance the anisotropy energy of grains, which accordingly results in the enhancement in switching field. Recent studies have shown that the 
higher-order anisotropy in a uniaxial grain effectively increases the energy barrier for magnetization reversal without any notable increase in the switching field [3]. Novel magnetic recording configuration utilizing tilted head fields or $45^{\circ}$ tilted media has been proved to be able to enhance the saturation recording capability of write head and minimize the effect of material property distribution on the SNR [2,4]. For application in high data-rate recording, the media are placed under high-speed switching fields, a good behavior of fast switching for the media is critically required. It is imperative to understand the switching mechanism of magnetization governing the upper limits of switching speed. Moreover, the magnetic fields from write heads generally deviate from the easy axis of the media. The effect of orientation distribution is crucial to the recording performance. Thus, the angular dependence of the high-speed switching behavior is very important. In this article, we study the effect of quartic anisotropy on the dynamic response of magnetization under a pulsed field applied at an arbitrary direction. The effects of magnetic-field pulse duration, strength, and orientation on the highspeed switching are investigated. The present results show that ultrafast switching is possible in the uniaxial particle with quartic anisotropy at an off-axis field well below the Stoner-Wohlfarth (SW) criterion.

\section{Model}

We consider a single-domain uniaxial particle whose magnetization $M$ reverses by coherent rotation. The long axis of an elongated particle is assumed to be coincident with the magnetocrystalline anisotropy axis, chosen to be in the $z$ direction. Therefore, the total energy density may be generally written as

$\varepsilon=K_{1} \sin ^{2} \theta+K_{2} \sin ^{4} \theta-\vec{M} \cdot \vec{H}_{\mathrm{a}}$.

Here $K_{1}$ and $K_{2}$ are the quadratic and quartic anisotropy constants, $\theta$ is the angle spanned by the particle's anisotropy axis and the magnetization $M,|M|=M_{\mathrm{s}}$, by assumption, where $M_{\mathrm{s}}$ is the saturation magnetization. $H_{\mathrm{a}}$ is the applied field, deviating from the $+z$ direction by an angle $\beta$. The time evolution of the magnetization under the influence of an effective magnetic field can be described by the Gilbert form of the Landau-Lifshitz (LLG) equation

$\frac{\mathrm{d} \vec{M}}{\mathrm{~d} t}=-\gamma \vec{M} \times \vec{H}_{\mathrm{eff}}+\frac{\alpha}{M_{s}} \vec{M} \times \frac{\mathrm{d} \vec{M}}{\mathrm{~d} t}$.

The constant $\gamma\left(=0.0176 \mathrm{Oe}^{-1} \mathrm{~ns}^{-1}\right)$ is the gyromagnetic ratio, $\alpha$ is the damping constant, and $H_{\text {eff }}$ is an effective magnetic field, defined as the sum of all fields acting on the magnetization [5]. The LLG equation keeps the magnitude of magnetization conserved and describes the relaxation of magnetization towards the effective field. The dissipation rate of energy depends on the damping constant, the effective field magnitude, and the orientation of magnetization with respect to the effective field. For convenience of simulations, the LLG equation can be easily expressed in the Cartesian coordinates and its solution can be readily found by numerical integration using the fourth-order Runge-Kutta method with double precision.

\section{Results and discussion}

For numerical calculations, the initial direction of magnetization is assumed along the $+z$ direction. The switching time is defined as the time taken for magnetization to first cross the hard plane after the application of external applied field. To keep things simple, we consider only an instantaneously applied field, without considering a rise time effect. Fig. 1 shows the variation of switching time versus applied field for field angles of $135^{\circ}$ and $175^{\circ}$ from the initial magnetization direction, respectively, which represent the switching properties within the fast or slowdown switching regimes separated by a critical field orientation (see Fig. 2). Clearly, the threshold of the dynamic switching field for the presence of $K_{2}$ is smaller than the SW criterion for small damping constants, as previous reports for a SW particle $\left(K_{2}=0\right)[5,6]$. For the field directions within the fast switching regime, the 'precessional' magnetization switching is dominated and the magnetization can cross over the hard plane during the first 

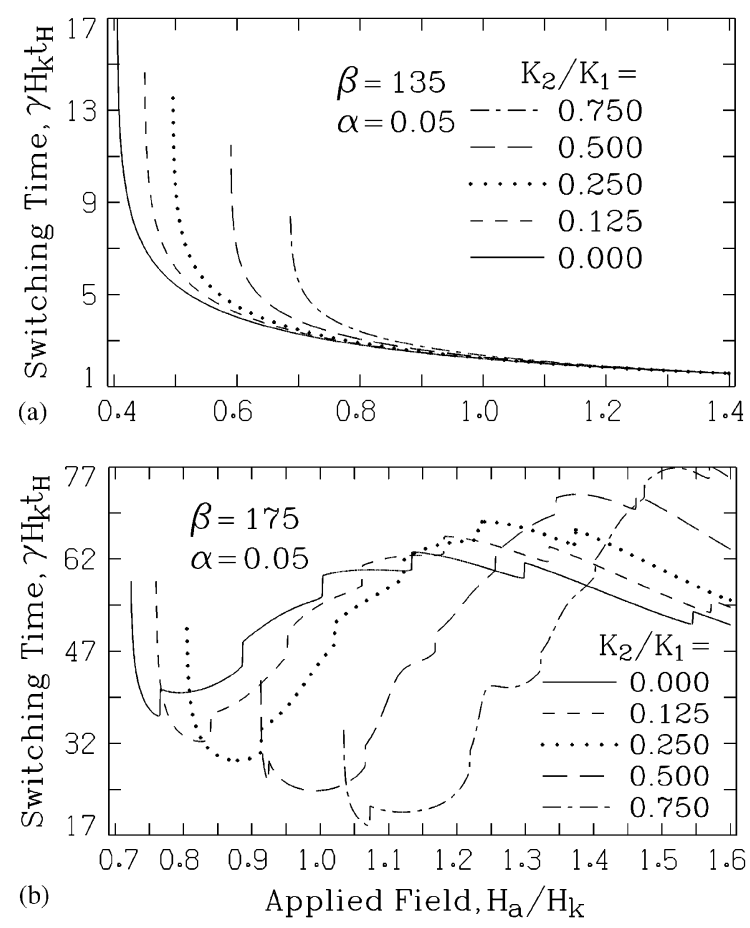

Fig. 1. The dependence of switching time on applied field magnitude for field angles at (a) $135^{\circ}$ and (b) $175^{\circ}$. Here $H_{\mathrm{k}}=$ $2 K_{1} / M_{\mathrm{s}}$.

precession cycle. The switching time is determined by the precession time [7]. Thus, the reversal process of magnetization is significantly fast and the switching time is very short. As the applied field strength increases, the increasing torque accelerates the precession of magnetization and the switching time decreases accordingly. For the applied field magnitude larger than the SW field, the energy of the particle is dominated by the Zeeman energy and the effective field is mainly from the applied field. Therefore, the switching time approaches to the timescale of precession period, the order of magnitude of $\left(\gamma H_{\mathrm{k}}\right)^{-1}$, and it can be empirically approximated as $A /\left(H_{\mathrm{a}}-H_{0}\right)$, where the symbols $A$ and $H_{0}$ are the adjustable parameters, depending on the field angle and the ratio of $K_{2} / K_{1}$ (Fig. 1(a)). Note that the effect of $K_{2}$ on the switching time is significant only for the applied field smaller than the anisotropy field $\left(H_{\mathrm{k}}=2 K_{1} / M_{\mathrm{s}}\right)$. As the field direction approaches the anisotropy axis, the magnetization during the

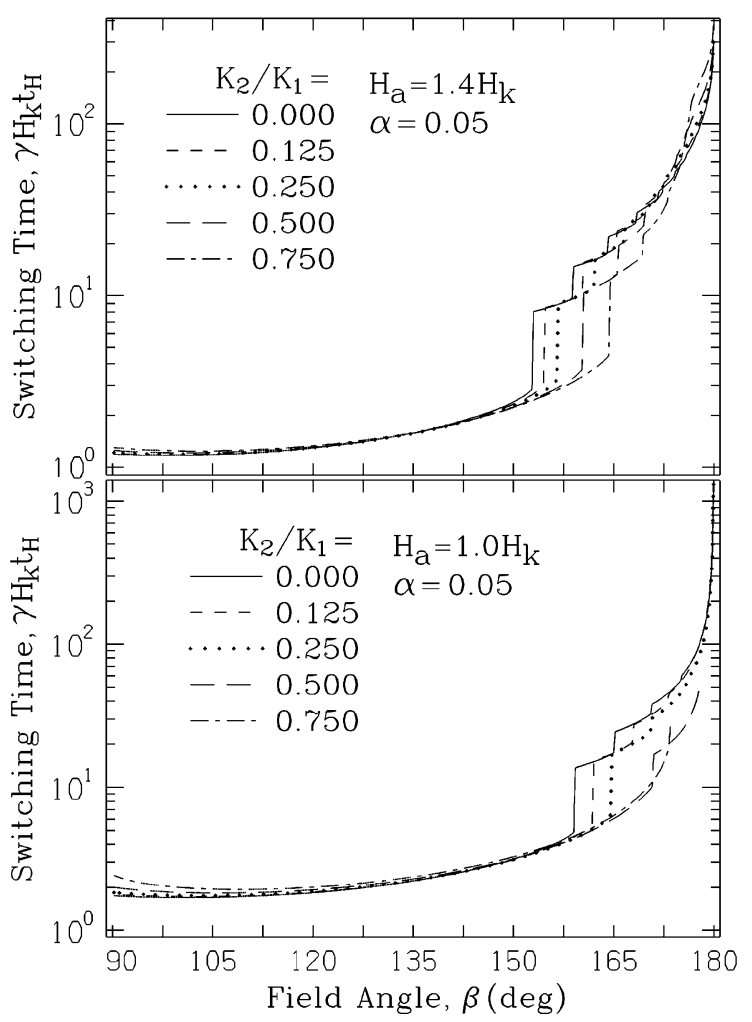

Fig. 2. The switching time versus applied field direction for several values of $K_{2} / K_{1}$. Here $H_{\mathrm{k}}=2 K_{1} / M_{\mathrm{s}}$.

reversal process may precess multiple cycles before crossing over the hard plane. The multiple-cycle precession of magnetization indeed makes the reversal process slow down, and hence a large switching time occurs, as seen in Fig. 1(b). Note that the switching time as a function of field strength does not behave monotonically but shows some step-like discontinuities in contrast to the results for the precession dominated case (cf. Fig. 1(a)). The slowdown reversal mode may account for the high switching field required for ultrashort duration of pulsed fields, as described below.

It is instructive to study the angular dependence of the switching time. As mentioned above, two distinct switching mechanisms can be clearly identified in Fig. 2. As the applied field is rotated away from the hard plane, the switching time shows a gradual increase up to a critical field direction, and then increases stepwise and rapidly as the field direction approaches the anisotropy 
axis. The ratio of switching times for the applied field along the two principal directions is up to about two orders of magnitude for the case of $H_{\mathrm{a}}=1.4 H_{\mathrm{k}}$. Decrease of the applied field magnitude generally increases the switching time due to the decreasing torque acting on the magnetization; however, if the value of the applied field is too small, below the threshold of dynamic switching field, no switching will appear, as seen for high $K_{2}$ at the field direction near the anisotropy axis. It is clear from Fig. 2 that decreasing the applied field magnitude or increasing the quartic anisotropy may expand the fast switching regime. Note that in the fast switching regime, the switching time is slightly affected by the presence of $K_{2}$.

When a pulsed field is applied, the final state is determined by the position of the magnetization as the field pulse is terminated. Therefore, reversal is possible if the magnetization crosses the hard plane at some point during its trajectory, even for the applied field magnitude below the SW field. The effect of field pulse duration $\left(t_{\mathrm{pf}}\right)$ on the switching field is studied, and the calculated results are shown in Fig. 3. The effect of $K_{2}$ obviously changes the angle-dependent behavior of dynamic switching field, which is distinctly different from the static results [8]. For long field pulses $\left(t_{\mathrm{pf}} \geqslant 100 / \gamma H_{\mathrm{k}}\right)$, the switching field is always smaller than the SW field for small damping constants, as expected. The difference between the dynamic switching field and the SW one increases with the increase in field angles relative to the anisotropy axis. Shortening the field pulse will lead to an increase in switching field, as expected. Specifically for the field direction near the anisotropy axis, the switching field is significantly enhanced relative to the SW field. In contrast, for the field direction within the fast switching regime the switching field is moderately enhanced as the field pulse duration shortens, down to the timescale of precession period $\left(t_{\mathrm{pf}} \approx 5 / \gamma H_{\mathrm{k}}\right)$. Obviously, the angular range of high switching field regions expands as the field pulse duration decreases. Fortunately, the range of high switching-field shrinks as the effect of $K_{2}$ comes into play, resulting in a broad minimum or a narrow switching field distribution around the field angle of $45^{\circ}$ for a large ratio of $K_{2} / K_{1}(\geqslant 0.75)$. The

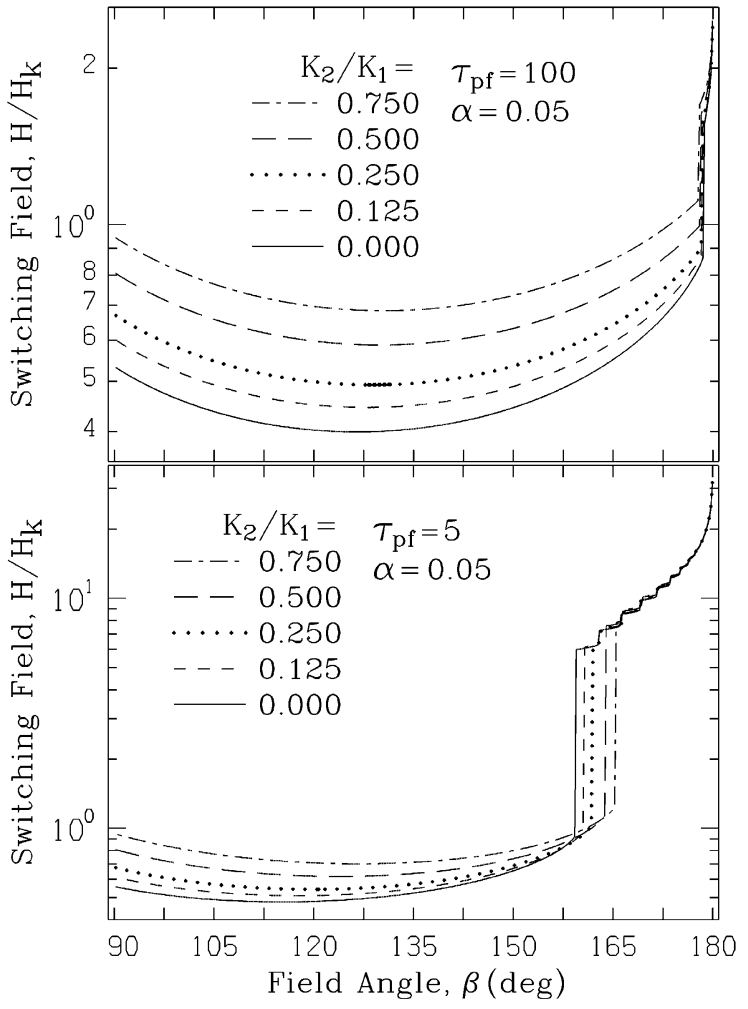

Fig. 3. The switching field versus applied field direction for several values of $K_{2} / K_{1}$. Here $\tau_{\mathrm{pf}}\left(=\gamma H_{\mathrm{k}} t_{\mathrm{pf}}\right)$ is the reduced duration of pulsed field and $H_{\mathrm{k}}=2 K_{1} / M_{\mathrm{s}}$.

reason may be that within the specific range of field angles, the energy landscape of the grain may be significantly modified by the quartic anisotropy and the precession easily drives the magnetization away from the initial direction, leading to a large reversal torque acting on the magnetization.

As described above, within a certain range of field angles, the quartic anisotropy can permit a significant reduction in both switching time and switching field of the uniaxial grain, compared to the case for a SW particle. The unusual property makes the grain exhibit fast switching behavior at the large field angles relative to the anisotropy axis. The present results suggest that to improve the high-speed switching property of the tilted media, the effect of higher-order anisotropy on the high-speed switching property of the uniaxial grain should be taken into account. Moreover, Uso and cowokers argued recently that a magnetic particle 
with uniaxial and combined (both uniaxial and cubic) type of magnetic anisotropy may have an optimal shape of energy barrier, consequently resulting in an enhanced gain, the ratio of the increase in effective energy barrier and the increase in the coercive force [9]. These findings suggest that for the development of future high-density recording media, it would be very important to control the higher-order magnetic anisotropy. Suitably adjusting the value of higher-order uniaxial and cubic anisotropies and tuning the tilted easy-axis angle may improve the recording characteristics of the media. It would be interesting to investigate the high-speed behavior for the novel recording media.

In summary, we have utilized the LLG equation to study the high-speed switching behavior for the uniaxial grain with quartic anisotropy. The present results show that for small damping constants and field pulse duration beyond the timescale of precession period, the dynamic switching field can be well below the SW criterion. The benefit from the quartic anisotropy is demonstrated by the good behavior of fast switching for large field angles relative to the anisotropy axis. This unique property of the uniaxial grain may be utilized to improve the extreme high density and ultrahigh data rate recording.

\section{Acknowledgments}

The authors are grateful to two anonymous reviewers whose valuable comments helped to improve the content of this paper. This work was supported by National Science Council of ROC under Grant no. NSC 92-2112-M019-005.

\section{References}

[1] M. Mallary, A. Torabi, M. Benakli, IEEE Trans. Magn. 38 (2002) 1719;

R. Wood, IEEE Trans. Magn. 36 (2000) 36; J.H. Judy, J. Magn. Magn. Mater. 235 (2001) 235.

[2] K.Z. Gao, H.N. Bertram, IEEE Trans. Magn. 39 (2003) 704

K.Z. Gao, H.N. Bertram, IEEE Trans. Magn. 38 (2002) 3675 .

[3] O. Kitakami, S. Satoni, N. Kikuchi, Y. Shimada, Jpn. J. Appl. Phys. 42 Part 2 (2003) L455;

H.N. Bertram, V.L. Safonov, Appl. Phys. Lett. 79 (2001) 4402.

[4] J.P. Wang, Y.Y. Zou, C.H. Hee, T.C. Chong, Y.F. Zhang, IEEE Trans. Magn. 39 (2003) 1930;

Y.Y. Zou, J.P. Wang, C.H. Hee, T.C. Chong, Appl. Phys. Lett. 82 (2003) 2473.

[5] Ching-Ray Chang, Jyh-Shinn Yang, Phys. Rev. B 54 (1996) 11057.

[6] K.Z. Gao, E.D. Boerner, H.N. Bertram, J. Appl. Phys. 93 (2003) 6549;

K.Z. Gao, E.D. Boerner, H.N. Bertram, Appl. Phys. Lett. 81 (2002) 4008;

L. He, W.D. Doyle, H. Fujiwara, IEEE Trans. Magn. 30 (1994) 4086.

[7] M. Bauer, J. Fassbender, B. Hilebrands, R.L. Stamps, Phys. Rev. B 61 (2000) 3410;

T. Devolder, C. Chappert, J. Phys. D: Appl. Phys. 36 (2003) 3115.

[8] Ching-Ray Chang, J. Appl. Phys. 69 (1991) 2431.

[9] N.A. Uso, C.-R. Chang, Z.-H. Wei, Appl. Phys. Lett. 83 (2003) 3749. 